\title{
Duodenal resections as part of the therapeutic strategy for duodenal malignant tumors
}

\author{
Nicolae BACALBASA ${ }^{1,2}$, Irina BALESCU ${ }^{3}$, \\ Adina CROITORU ${ }^{4,5}$, Simona DIMA ${ }^{4}$, \\ Mihaela VILCU ${ }^{1,2}$, Iulian BREZEAN ${ }^{1,2}$ \\ 1 "Carol Davila" University of Medicine and Pharmacy, Bucharest, Romania \\ 2 "Ion Cantacuzino" Clinical Hospital, Bucharest, Romania \\ ${ }^{3}$ Ponderas Academic Hospital, Bucharest, Romania \\ ${ }^{4}$ Fundeni Clinical Institute, Bucharest, Romania \\ ${ }^{5}$ Faculty of Medicine, "Titu Maiorescu" University, Bucharest, Romania
}

\begin{abstract}
Duodenal carcinoma remains a very rare pathology, the most efficient therapeutic strategy remaining intensively debatable. However, it seems that the most appropriate therapeutic approach for each case can be established depending on the dimensions and stage of the tumor and according to the tumoral localization. Although initially it has been proposed that in all cases in which resection is feasible a duodenopancreatectomy should be performed, nowadays the therapeutic strategy has been submitted to permanent changes, more conservative approaches such as endoscopic full thickness resection or pancreas preserving duodenal resection being proposed with encouraging results. This is a literature review of the largest studies which investigated the feasibility of duodenal resections for duodenal malignant tumors.
\end{abstract}

Keywords: duodenal malignancies, duodenal resection, complications, survival

\section{INTRODUCTION}

Duodenal cancer is an uncommon disease, representing up to $40 \%$ of all small bowel malignant tumors; in consequence, most authors which investigate this pathology include it in the same studies which also refer to periampulary tumors or other small bowel malignant lesions (1).

Due to the small number of cases there is a permanent debate in regard to the most appropriate therapeutic strategy, to the most significant prognostic factors as well as to the indications and type of adjuvant therapy (1-4). When it comes to the most appropriate surgical strategy, another important issue is related to the resectability of the lesion; unfortunately an important number of cases are diagnose in advanced stages of the disease, when locally invasion involving certain unresectable structures is already present; in such cases surgery has only a palliative intent and consist of creating an internal bypass in order to alleviate the symptoms caused by the digestive stenosis.

\section{Surgical strategies in patients with duodenal malignant tumors}

Depending on the dimensions and location of the duodenal malignant process, different therapeutic strategies have been proposed; therefore, while small, superficial tumors can be safely resected via an endoscopic approach, larger lesions will certainly need performing a more complex 
procedure such as pancreas preserving duodenal resection or even pancreatico-duodenectomy (1). Cases presenting small duodenal tumors can be also submitted to endoscopic full thickness resection under laparoscopic observation; in this manner any incident which might take place during endoscopy can be rapidly identified and solved (5).

Special care should be taken in cases in which the tumor is developed at the level of D2 duodenum; in such cases certain authors propose performing a duodenotomy followed by direct visualisation of the tumor and its' relationship with the ampulla rather than intraoperative endoscopy or cannulation (6). However, irrespectively of the tumoral localisation, decision of performing a duodenal limited resection should be taken only after excluding the tumoral pancreatic involvement.

\section{Pancreatic preserving duodenal resections for duodenal adenocarcinoma}

This type of procedure is an extremely demanding one and is strongly influenced by the localization and size of the tumoral process. While for cases diagnosed with D3 or D4 tumors most authors agree to perform a limited to duodenum resection whenever the size of the lesion permit it, for lesions located at the level of D1 and D2 there are permanent controversies regarding the benefits of a limited resection (7-9).

In the study conducted by Cecchini et al. and published in 2012 the authors included 169 patients diagnosed with duodenal adenocarcinoma between 1982 and 2010 (1); among these cases, a potential radical resection was feasible in only 103 cases, the unresectable lesions being most often located at the level of D3 duodenum. As for the type of resection, it consisted of segmental duodenal resection in 14 cases, the remaining 87 cases being submitted to pancreaticoduodenectomy. However, there was no significant difference in terms of postoperative complications, length of hospital in stay or postoperative mortality between the two types of surgery. When it comes to the long term results, the univariate analysis demonstrated that the most important prognostic factors impacting on the overall survival rates were related to the lymph node involvement, lymph node ratio, stage of the tumor and presence of perineural invasion; in the meantime in multivariate analysis the presence of perineural invasion represented the most powerful predictive factor for survival. Moreover, the analysis of recurrence patterns also demonstrated that the presence of the perineural invasion was the strongest predictive factor for relapse; however, it seems that the type of resection (duodenal resection versus pancreaticoduodenctomy) did not influence the long term outcomes (1).

One of the largest studies which aimed to compare the impact on the long term outcomes of the type of resection (duodenal resection versus pancreatoduodenectomy) was conducted by Jorda Cloyd et al. and was published in 2015 in Annals of Surgical Oncology journal (10). The study included 1611 cases diagnosed with duodenal adenocarcinomas submitted to surgery between 1988 and 2010 in Stanford University; among these cases there were 865 patients submitted to pancreatoduodenectomy - considered as radical resections and 746 cases submitted to duodenal resections - considered as simple resections; however, patients submitted to radical resections were more likely to present larger, more biologically aggressive tumors. Therefore, patients submitted to radical resections benefited from a significantly higher number of retrieved nodes while the median number of positive retrieved nodes was also significantly higher. However, there was no significant difference in terms of postoperative complications, while the long term outcomes-described by the disease free survival and overall survival were similar between the two groups. Moreover, when stratifying patients according to the stage of disease, there was no significant difference in terms of survival based on the type of resection (10). An interesting observation was the one regarding the extent of the lymph node dissection and the impact on survival: although patients submitted to radical resections benefited from a more extended lymph node dissection and a higher number of positive retrieved nodes, there was no significant difference in terms of survival between this group and the group submitted to simple resections. This aspect was explained by the authors by the fact that the first and fourth portions of the duodenum probably drain into the lymphatic stations from the pylorus and from the mesenteric root and not at the level of the pancreatic head stations (10).

However, not all studies conducted on this theme came to demonstrate the comparable outcomes of these two procedures; for example, in the study conducted by Sohn et al. and published in 1998 the authors included 35 patients submitted to pancreatoduodenectomy and 13 cases submitted to segmental resections and demonstrated that the five year overall survival rate was of $63 \%$ among the first group and 0 for the second group; however, this significant difference in terms of sur- 
vival was rather caused by the fact that among patients submitted to duodenal resections there were $23 \%$ cases with positive resectional margins, while this per cent was of only $3 \%$ among cases submitted to pancreatoduodenectomy. Interestingly, patients submitted to pancreas preserving duodenal resections experienced a lower rate of early postoperative complications (2).

An interesting study which included this time exclusively patients submitted to segmental duodenal resections was conducted by Dorcaratto et al. and was published in 2015 (6); the study included 11 patients submitted to duodenal resections between January 2007 and December 2013 at St Vincent University Hospital, Dublin, Ireland. The most commonly resected duodenal part was segment 3 (in seven cases), followed by segment 2 and 4 (each in four cases) and segment 1 (in two cases); moreover, in seven cases two or more segments were resected. In nine cases reconstruction was performed by using a duodeno-duodenal anastomosis or a duodeno-jejunal anastomosis while in two cases involving D1 or D2 a Roux en Y reconstruction was the option of choice. In all cases in which D2 resection was needed the ampulla was identified by direct visualization; the median length of surgery was of 191 minutes, no intraoperative complciations being reported. Postoperatively the authors reported two cases of serious complications - an anastomotic leak and a bleeding - both cases being managed in a conservative manner; when it comes to the completeness of resection, a radical procedure was achieved in all cases, all patients presenting negative resection margins. During the follow-up period, a single patient experienced recurrent disease and died seven months later. These data came to demonstrate once again the safety, feasibility and efficacy of the procedure when performed in highly specialized centers (6).

A recent meta-analysis conducted by Meijer et al. and published in 2018 was conducted on 26 observational studies and 6438 patients diagnosed with duodenal adenocarcinomas, $71 \%$ being submitted to surgery with radical intent (11). The authors underlined the fact that both segmental duodenal resections and pancreatoduodenectomy were associated with similar rates of long term survival; in the meantime the accuracy of the lymph node dissection was similar for the two types of surgery. In the meantime, the most important prognostic factor was identified to be the status of the retrieved lymph nodes while the as- sociation of any type of adjuvant therapy after curative resection did not seem to impact the long term outcomes (11).

In order to minimize the perioperative morbidity, the Japanese authors conducted by Yanagimoto et al. reported a novel laparoscopic and endoscopic surgical technique which has been successfully performed so far in 10 cases (12). The procedure begins in a laparoscopic manner by freeing the transverse mesocolon and exposing the duodenum, performing a Kocher maneuver and identifying the duodenal lesion. From this moment the endoscopic phase of the procedure begins by identifying the tumor and performing a submucosal endoscopic resection. The procedure is ended by a laparoscopic suture of the resulting duodenal defect using a seromuscular layer. The method has been successfully performed in all cases between March 2015 and March 2017, the median diameter of the resected specimen being of $41 \mathrm{~mm}$, all specimens presenting negative resection margins. There was no reported serious complication (higher than grade 2 complications according to Clavien Dindo system) while the median length of hospital in stay was of 9 days. Moreover, after a median follow up period of 15 months there was no recurrent disease. When it comes to the histopathological type of the tumor, there were six cases of duodenal adenocarcinomas, three adenomas and one neuroendocrine tumor (12).

\section{CONCLUSIONS}

Performing an isolated duodenal resection or a full thickness resection for duodenal malignant lesions represents demanding procedures which might associate an important risk of developing postoperative complications which should be performed only in specialised centers. However, these types of procedures permit the entire preservation of the pancreatic head and avoid submitting the patient to an unnecessary pancreatic resection.

\section{Acknowledgement}

This work was supported by the project entitled „Multidisciplinary Consortium for Supporting the Research Skills in Diagnosing, Treating and Identifying Predictive Factors of Malignant Gynecologic Disorders", project number PN-III-P1-1.2PCCDI2017-0833.

Conflict of interest: none declared 


\section{REFERENCES}

1. Cecchini S, Correa-Gallego C, Desphande $V$ et al. Superior prognostic importance of perineural invasion vs. lymph node involvement after curative resection of duodenal adenocarcinoma. J.Gastrointest. Surg. 2012; 16: 113-20.

2. Ryder NM, Ko CY, Hines OJ et al. Primary duodenal adenocarcinoma: a 40-year experience. Arch.Surg. 2000; 135: 1070-4.

3. Sohn TA, Lillemoe KD, Cameron JL et al. Adenocarcinoma of the duodenum: factors influencing long-term survival. J.Gastrointest.Surg. 1998; 2: 79-87.

4. Tocchi A, Mazzoni G, Puma F et al. Adenocarcinoma of the third and fourth portions of the duodenum: results of surgical treatment. Arch.Surg. 2003; 138: 80-5.

5. Tsujimoto $\mathrm{H}$, Ichikura $\mathrm{T}$, Nagao $\mathrm{S}$ et al. Minimally invasive surgery for resection of duodenal carcinoid tumors: endoscopic full-thickness resection under laparoscopic observation. Surg.Endosc. 2010; 24: 471-5.

6. Dorcaratto D, Heneghan HM, Fiore B et al. Segmental duodenal resection: indications, surgical techniques and postoperative outcomes. J.Gastrointest.Surg. 2015; 19: 736-42.

7. Agrawal S, McCarron EC, Gibbs JF et al. Surgical management and outcome in primary adenocarcinoma of the small bowel. Ann.Surg. Oncol. 2007; 14: 2263-9.

8. Bakaeen FG, Murr MM, Sarr MG et al. What prognostic factors are important in duodenal adenocarcinoma? Arch.Surg. 2000; 135: 635-41.

9. Bucher P, Gervaz P, Morel P. Long-term results of radical resection for locally advanced duodenal adenocarcinoma. Hepatogastroenterology 2005; 52: 1727-9.
10. Cloyd JM, Norton JA, Visser BC et al. Does the extent of resection impact survival for duodenal adenocarcinoma? Analysis of 1,611 cases. Ann.Surg. Oncol. 2015; 22: 573-80.

11. Meijer LL, Alberga AJ, de Bakker JK et al. Outcomes and Treatment Options for Duodenal Adenocarcinoma: A Systematic Review and Meta-Analysis. Ann.Surg. Oncol. 2018; 25: 2681-92.

12. Yanagimoto $\mathrm{Y}$, Omori $\mathrm{T}$, Jeong-Ho $\mathrm{M}$ et al. Feasibility and Safety of a Novel Laparoscopic and Endoscopic Cooperative Surgery Technique for Superficial Duodenal Tumor Resection: How I Do It. J.Gastrointest.Surg. 2019. 